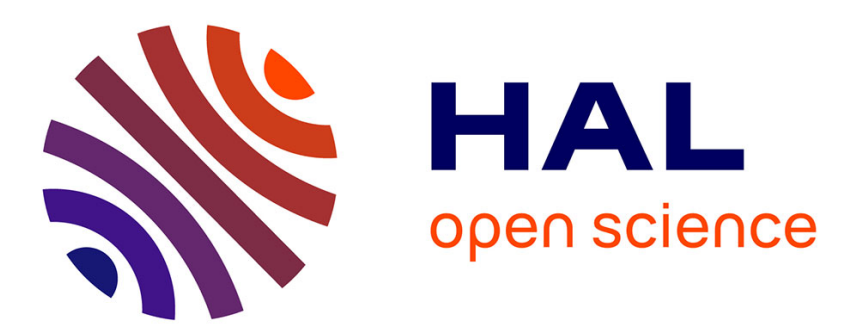

\title{
A new prediction scheme for input delay compensation in restricted-feedback linearizable systems
}

William Pasillas-Lépine, Antonio Loria, Trong-Bien Hoang

\section{To cite this version:}

William Pasillas-Lépine, Antonio Loria, Trong-Bien Hoang. A new prediction scheme for input delay compensation in restricted-feedback linearizable systems. IEEE Transactions on Automatic Control, 2016, 61 (11), pp.3693-3699. 10.1109/TAC.2016.2531042 . hal-01357238

\section{HAL Id: hal-01357238 \\ https://hal.science/hal-01357238}

Submitted on 5 Mar 2020

HAL is a multi-disciplinary open access archive for the deposit and dissemination of scientific research documents, whether they are published or not. The documents may come from teaching and research institutions in France or abroad, or from public or private research centers.
L'archive ouverte pluridisciplinaire $\mathbf{H A L}$, est destinée au dépôt et à la diffusion de documents scientifiques de niveau recherche, publiés ou non, émanant des établissements d'enseignement et de recherche français ou étrangers, des laboratoires publics ou privés. 


\section{A new prediction scheme for input delay compensation in restricted-feedback linearizable systems}

William Pasillas-Lépine $^{1} \quad$ Antonio Loría $^{1} \quad$ Trong-Bien Hoang ${ }^{2}$

\begin{abstract}
The input-output inversion of a system under the effect of input delays typically relies on the ability to predict the future of the system's state. Indeed, if the latter is known ahead of time, one can cope with the input delay by using a prediction of the state instead of the state itself. Such methods are efficient when the plant is stable but become numerically unstable otherwise. We present a new method to compensate input delays; our approach relies on imposing a desired error dynamics which is designed to be linear and asymptotically stable at the origin. Then, the state prediction is computed from the state reference trajectory and the predicted error dynamics. In this paper we concentrate on the case study of systems in strict feedback form and present a simple backstepping procedure.
\end{abstract}

\section{INTRODUCTION}

Input-delay compensation for controlled systems often relies on the design of a predictor, $z(t)$, that estimates the future of the system's state $x(t)$ one delay $h$ ahead. That is, such that $z(t)=x(t+h)$. The simplest example is probably that of the Smith predictor [1]. Designed for stable linear systems and based on frequency domain techniques, this method is widely used in industrial applications. For unstable linear systems other solutions based on a state-space representation are available (see, e.g., [2] and [3]).

The predicted value of the state, $z(t)$, is typically constructed by integrating the system's dynamics along trajectories. Consider, for example, the stabilization problem for the linear system

$$
\dot{x}(t)=A x(t)+B u(t-h), \quad x \in \mathbb{R}^{n},
$$

A classical prediction function (see, e.g., [2] and [3]) is

$$
z(t)=x(t)+\int_{t-h}^{t} e^{A(t-h-s)} B u(s) d s,
$$

which satisfies the differential equation

$$
\dot{z}(t)=A z(t)+e^{-A h} B u(t) .
$$

Provided that $(A, B)$ is stabilizable, there exists $K$ such that $u=$ $-K z(t)$ stabilizes (3). Nevertheless, the numerical implementation of such prediction techniques may lead to an unstable behavior (see, e.g., [4] and [5]), at least when the original system is unstable. In [5] the authors identified that this instability mechanism is related to the occurrence of unstable eigenvalues with arbitrarily large imaginary parts and gave conditions for stability of the closed-loop system using a filtered control input. At the opposite, when the original system is both linear and stable, a recent result [6] shows that such schemes admit a stable numerical implementation.

It is only recently that methods have been proposed for several classes of nonlinear systems (see, e.g., [7], [8], [9], [10], [11], [12] and the references therein). In this paper we present a completely different prediction method. Owing to the fact that our aim is to find a control law that tracks a given reference for the system's output, we consider the error dynamics as a part of the control design by imposing a reference error model. Then, the predictor is designed based on the integration of the target error dynamics, which is stable by design, in contrast to the possibly unstable plant dynamics. Indeed,

${ }^{1}$ W. Pasillas-Lépine and A. Loría are with CNRS. Address: L2S-Supélec, 3 rue Joliot-Curie, 91192 Gif-sur-Yvette, France. Corresponding author: pasillaselss. supelec.fr.

${ }^{2}$ T. B. Hoang is with L2S-Université Paris Sud-Supélec, 3 rue Joliot-Curie, 91192 Gif-sur-Yvette, France. one can always chose a tracking error dynamics that is both stable and linear, at least asymptotically. The evolution of such a system can be predicted at ease; based on the predicted error and the predicted reference values, we compute a prediction of the state itself.

Next, our certainty equivalence control law (obtained by replacing the unknown future of the state by its prediction) is tailored to obtain the target closed-loop system, modulo a vanishing perturbation. The latter results from the prediction error. The stability analysis is also original: it relies on the ability to separate the tracking error dynamics from that corresponding to the prediction error. We show that the overall closed-loop system has a cascaded structure and present original results on stability of cascaded systems.

We apply our novel prediction-based control method to a class of systems that can be linearized using a change of coordinates and a restricted-feedback transformation [13],

$$
\left\{\begin{aligned}
\dot{x}_{1}(t) & =f_{1}\left(x_{1}(t)\right)+x_{2}(t) \\
& \vdots \\
\dot{x}_{n-1}(t) & =f_{n-1}\left(x_{1}(t), \ldots, x_{n-1}(t)\right)+x_{n}(t) \\
\dot{x}_{n}(t) & =f_{n}(x(t))+u(t-h),
\end{aligned}\right.
$$

where $x(t):=\left[\begin{array}{lll}x_{1}(t) & \cdots & x_{n}(t)\end{array}\right]^{\top} \in \mathbb{R}^{n}$ and $y(t)=x_{1}(t)$ is the system's output, for which we have a reference $y^{*}(t)$. Furthermore, conditions for the existence of a global transformation into this triangular form are available (see [14] and [15]).

We solve the stabilization problem for (4), following a classical backstepping rationale that is, we consider $x_{i}$ as a virtual control input to the $x_{i-1}$-dynamics and a reference trajectory for the $x_{i}$ dynamics in the presence of constant input delays. As previously explained, the novelty of our results lays in the fact that we stand away from the classical paradigm of integrating the system's state.

Observe that more general classes of systems have been considered before (see, e.g., [8], [9], and [10]), but with different control objectives and prediction methods that lead to different control laws. Additionally, for a similar class of systems, a different prediction scheme has been proposed recently in [16] and [17] (see also [18]). Some readers may also find links between our approach and the methods proposed in [19] and [20], in order to construct state predictors (see also [21]). One should stress, moreover, that the stability conditions proposed in Theorem 1 and 2 are not necessarily sharp (in a linear context, the works [22] and [23] give sharper stability conditions).

The rest of the paper is organized as follows. In Section II we present our prediction method and state our main result, whose the proof is based on a stability result for cascaded systems of delayed functional differential equations, originally presented in Section III. We conclude with some remarks in Section IV and with some technical proofs, which are included in the Appendix.

Notation: For a diagonal matrix $\beta$ we use $\beta_{\min }$ and $\beta_{\max }$ to denote, respectively, its smallest and largest elements. For $t_{\circ} \in \mathbb{R}_{\geq 0}$ and any absolutely continuous $\phi:[0, h] \rightarrow \mathbb{R}^{n}$, the solutions of a functional differential equation

$$
\dot{z}(t)=f(t, z(t), z(t-h)), \quad \forall t \geq t_{\circ},
$$

with $f$ locally Lipschitz in $z$, uniformly in $t$, and locally integrable in $t$, are absolutely continuous functions that satisfy, additionally to (5), the initial condition

$$
z\left(t_{\circ}-s\right)=\phi(s), \quad \forall s \in[0, h] .
$$

We say that the trivial solution $z(t) \equiv 0$ is globally exponentially stable if there exist $\kappa, \lambda>0$ such that, for any absolutely continuous 
initial condition $\phi$,

$$
|z(t)| \leq \kappa\|\phi\| \mathrm{e}^{-\lambda\left(t-t_{\circ}\right)}, \quad \forall t \geq t_{\circ},
$$

where

$$
\|\phi\|:=\left(|\phi(0)|^{2}+\sup _{s \in[0, h]}|\phi(s)|^{2}\right)^{1 / 2} .
$$

\section{THE PREDICTION METHOD}

\section{A. Scalar systems}

To better explain our method, let us start with the tracking problem for a scalar nonlinear system,

$$
\dot{x}(t)=f(x(t))+u(t), \text { for } x(t) \in \mathbb{R},
$$

in the absence of input delays. This is a trivial task. Indeed, if we want $x(t)$ to converge towards a continuously differentiable reference $x^{*}(t)$, we may define the tracking error

$$
e(t)=x(t)-x^{*}(t)
$$

and apply the linearizing control input

$$
u(t)=-f(x(t))-\alpha e(t)+\dot{x}^{*}(t),
$$

which stabilizes the origin of the error dynamics

$$
\dot{e}(t)=-\alpha e(t)
$$

globally and exponentially, for any control gain $\alpha>0$.

In the presence of an input delay, that is for a system described by the functional differential equation

$$
\dot{x}(t)=f(x(t))+u(t-h), \text { for } x(t) \in \mathbb{R},
$$

this task is more involved since the previous control input leads to the error dynamics

$\dot{e}(t)=-\alpha e(t-h)+f(x(t))-f(x(t-h))+\dot{x}^{*}(t-h)-\dot{x}^{*}(t)$,

as opposed to the "ideal" error dynamics (10).

One way to compensate the delay is to use, if possible at all, the future values of $\dot{x}^{*}$ and $x$, at the instant $t+h$. Nevertheless, on one hand, in a number of applications the reference trajectory is unknown in advance, e.g., in the case when a human operator fixes it in realtime. This justifies to redefine the control goal to tracking the delayed reference, i.e., to make

$$
\lim _{t \rightarrow \infty} e(t)=0, \quad \text { for } \quad e(t):=x(t)-x^{*}(t-h),
$$

instead of (8). On the other hand, in lack of $x(t+h)$, we introduce a state prediction, which we denote by $x^{P}(t, h)$ and, we apply the certainty equivalence control input

$$
u(t)=-f\left(x^{P}(t, h)\right)-\alpha e(t)+\dot{x}^{*}(t),
$$

instead of (9), so that in closed loop with (11) we have

$$
\dot{e}(t)=-\alpha e(t-h)+f(x(t))-f\left(x^{P}(t-h, h)\right)
$$

where $x^{P}(t-h, h)$ corresponds to the prediction of $x(t)$, made at the instant $t-h$. Clearly, if the state prediction $x^{P}$ is perfect the error dynamics becomes

$$
\dot{e}(t)=-\alpha e(t-h),
$$

whose origin is known to be exponentially stable if $0<\alpha<\pi / 2 h$. Otherwise, the last two terms in (14) induce a prediction bias $p(t)$ that is,

$$
p(t):=x^{P}(t-h, h)-x(t) .
$$

Notice that if we design the prediction bias to vanish asymptotically and the solutions remain bounded one may use a vanishingperturbation argument to conclude convergence of the estimation errors. To better see this, notice that the closed-loop equation (14) may be re-written as

$$
\dot{e}(t)=-\alpha e(t-h)+f(x(t))-f(x(t)+p(t)) .
$$

Thus, we regard (15) as a target error dynamics.

It is based on these observations that we design the state predictor, but in contrast to most available methods in the literature, which rely on the integration of the system's dynamics, ours is based on the definition of the prediction error. This is obtained by integrating the stable target error dynamics (15), modulo the addition of an integral term in order to damp the perturbation induced by the prediction bias, i.e., we define the error prediction as

$$
e^{P}(t, s):=e(t)-\alpha \int_{t}^{t+s} e(\tau-h) d \tau-\beta \int_{-\infty}^{t+s} p(\tau-h) d \tau,
$$

for $s \in[0, h]$ while the prediction bias is naturally computed by evaluating the difference between the tracking error measured at the instant $t$ and its prediction made $h$ units of time earlier, i.e.,

$$
p(t)=e^{P}(t-h, h)-e(t) .
$$

Correspondingly, we define the estimate of the future values of the system's state based on the estimation error (17), i.e.,

$$
x^{P}(t, s):=x^{*}(t+s-h)+e^{P}(t, s), \quad \forall s \in[0, h]
$$

that is, the term $x^{P}(t, h)$ used in the control law in (13) depends on the reference value $x^{*}(t)$ and the error prediction.

Remark $1 \mathrm{We}$ stress that the implementation of our prediction scheme is straightforward: to estimate its future values $e^{P}(t, s)$, for $s \in[0, h]$, only the past values of the error $e(t-s)$ are needed. Even though this requires to store the past values (for all $s \in[t-h, t]$ ) of all variables in a memory buffer, this potential drawback is compensated by its numerical stability.

Next, for the purpose of analysis, we compute the dynamics of $p(t)$. To that end, we differentiate on both sides of (18), we use (14) and (17) and, to compact the notation, we introduce

$$
\psi(s):=f(x(s))-f(x(s)+p(s)) .
$$

Then, considering (18), (17) at $t-h$ with $s=h$, and (14) we obtain

$$
\dot{p}(t)=-\beta p(t-h)-\psi(t)+\psi(t-h) .
$$

A useful property of this equation is that under a Lipschitz condition on $f$ one may use Lyapunov-Krasovskiî's method to establish exponential stability of $\{p=0\}$ for sufficiently large $\beta$; this result is global if so is the Lipschitz property. As a matter of fact, it may also be shown that (16) is input-to-state-stable from the input $p(t)$. Thus, together with (16), Equation (20) forms a closed-loop system that consists in the cascade of two exponentially stable systems. This leads to the following statement, whose proof is a direct consequence of our main result (see Theorem 1 further below).

Proposition 1 Consider the scalar input-delay system (11). Assume that there exists $\gamma$ such that the function $f$ satisfies

$$
|f(x)-f(y)| \leq \gamma|x-y|, \quad \forall x, y \in \mathbb{R} .
$$

For any given $h^{*}>0$, if the gains $\alpha$ and $\beta$ satisfy the relations

$$
\alpha<1 / h^{*} \text { and } \beta \geq(9 / 4) \gamma+\beta(\beta+2 \gamma) h^{*},
$$

the origin of the closed-loop system, given by (11) with the control $u(t)$ defined by (13) and (17)-(19), is globally exponentially stable for any constant delay $h \in\left[0, h^{*}\right]$. 
Observe that the constraint on $\beta$ imposed by condition (21) is sufficient for the exponential stability of $\{p=0\}$, for (20). Additionally, in the absence of the nonlinearities, one may take $\gamma=0$ and, hence, for the system $\dot{p}(t)=-\beta p(t-h)$ we obtain $\beta<1 / h^{*}$.

\section{B. Triangular systems}

We now show how the prediction algorithm previously explained for scalar systems may be used recursively to design input-delay compensation controllers for systems in triangular form (4). Firstly, since the control input is subject to a constant delay $h$, as for the first-order counterpart of (4), the control goal is set to following a delayed reference. That is, we define

$$
e_{i}(t)=x_{i}(t)-x_{i}^{*}(t-h), \quad \text { for } 1 \leq i \leq n .
$$

Then, following the classical backstepping procedure, the variable $x_{i+1}$ is viewed as a virtual control input to each $\dot{x}_{i}$-equation in (4) so, analogously to (13), we define

$$
x_{i}^{*}(t):=-f_{i-1}\left(\bar{x}_{i-1}^{P}(t, h)\right)-\alpha_{i-1} e_{i-1}(t)+\dot{x}_{i-1}^{*}(t),
$$

for $2 \leq i \leq n$, where $\bar{x}_{i}^{P}:=\left[x_{1} \cdots x_{i}\right]^{\top}$; and $x_{1}^{*}(t):=y^{*}(t)$. The terms $x_{i}^{P}(t, h)$, for $1 \leq i \leq n$, denote the predictions of $x_{i}(t+h)$ computed at the instant $t$ and we show farther below how they are computed.

Now, from (22) we have $x_{i+1}(t)=e_{i+1}(t)+x_{i+1}^{*}(t-h)$ so, using (23), we see that the $i$ th equation in (4) is equivalent to

$$
\dot{e}_{i}(t)=-\alpha e_{i}(t-h)+e_{i+1}(t)+f\left(\bar{x}_{i}(t)\right)-f_{i}\left(\bar{x}_{i}^{P}(t-h, h)\right)
$$

for $2 \leq i \leq n-1$. Correspondingly, for $i=n$, the control law is defined as

$$
u(t)=-f_{n}\left(x^{P}(t, h)\right)-\alpha_{n} e_{n}(t)+\dot{x}_{n}^{*}(t) .
$$

When the prediction of the state $x^{P}$ is perfect, the error dynamics has the convenient cascaded structure

$$
\begin{aligned}
\dot{e}_{1}(t) & =-\alpha_{1} e_{1}(t-h)+e_{2}(t) \\
\dot{e}_{2}(t) & =-\alpha_{2} e_{2}(t-h)+e_{3}(t) \\
& \vdots \\
\dot{e}_{n-1}(t) & =-\alpha_{n-1} e_{n-1}(t-h)+e_{n}(t) \\
\dot{e}_{n}(t) & =-\alpha_{n} e_{n}(t-h),
\end{aligned}
$$

which is regarded as the target error dynamics. Hence, the controller and the predictor are defined with the aim that the error dynamics correspond to (25). Note, moreover, that the latter consists in a chain of input-to-state stable systems, driven by the $n$-th system, whose origin is exponentially stable. This is the rationale which leads to the design of the predictor.

As in the scalar case, for $i=n$, the prediction error is computed as

$$
e_{n}^{P}(t, h)=e_{n}(t)-\alpha_{n} \int_{t}^{t+h} e_{n}(\tau-h) d \tau-\beta_{n} \int_{-\infty}^{t+h} p_{n}(\tau-h) d \tau
$$

where

$$
p_{n}(t)=e_{n}^{P}(t-h, h)-e_{n}(t) .
$$

Then, we use the latter to compute recursively all other prediction errors, from $i=n-1$ down to 1 , defining

$$
\begin{aligned}
e_{i}^{P}(t, h)= & e_{i}(t)-\alpha_{i} \int_{t}^{t+h} e_{i}(\tau-h) d \tau-\beta_{i} \int_{-\infty}^{t+h} p_{i}(\tau-h) d \tau \\
& +\int_{t}^{t+h} e_{i+1}^{P}(\tau-h, h) d \tau
\end{aligned}
$$

and using the latter, we compute the estimate of $x_{i}(t+h)$ at the instant $t$, as

$$
x_{i}^{P}(t, h)=x_{i}^{*}(t)+e_{i}^{P}(t, h)
$$

which is needed in (23) -see also (24).

We are ready to present our main result.

Theorem 1 Consider the restricted-feedback linearizable system (4) and assume that, for each $1 \leq i \leq n$, there exists $\gamma_{i}$ such that

$$
\left|f_{i}(z)-f_{i}(y)\right| \leq \gamma_{i}|z-y|, \quad \forall z, y \in \mathbb{R}^{i} .
$$

Then, given any $h^{*}>0$, if the control gains $\alpha:=\operatorname{diag}\left\{\alpha_{1} \cdots \alpha_{n}\right\}$ and $\beta:=\operatorname{diag}\left\{\beta_{1} \cdots \beta_{n}\right\}$ satisfy

$$
\begin{aligned}
\alpha_{\min } & \geq 4+\alpha_{\max }\left(\alpha_{\max }+2\right) h^{*} \\
\beta_{\min } & \geq 4 \gamma+\beta_{\max }\left(\beta_{\max }+2 \gamma\right) h^{*},
\end{aligned}
$$

where $\gamma:=\max \left\{\gamma_{i}\right\}+1$, the origin of the closed-loop system, given by (4) with the controller defined by (24) and (26)-(29), is globally exponentially stable for any constant delay $h \in\left[0, h^{*}\right]$.

The proof relies on the observation that the closed-loop equations have a cascaded form in which the error dynamics (25), whose origin is exponentially stable by design, is perturbed by the prediction bias $p(t)$. To see this, we proceed to compute the derivatives of $e(t)$ and $p(t)$ generated by the closed-loop dynamics. We start by introducing a compact notation, defining $\bar{p}_{i}:=\left[p_{1} \cdots p_{i}\right]^{\top}, \bar{p}_{n}=p$, and

$$
\psi_{i}(s):=f_{i}\left(\bar{x}_{i}(s)\right)-f_{i}\left(\bar{x}_{i}(s)+\bar{p}_{i}(s)\right),
$$

so that, for each $1 \leq i \leq n$,

$$
\dot{e}_{i}(t)=-\alpha_{i} e_{i}(t-h)+e_{i+1}(t)+\psi_{i}(t) .
$$

Next, we differentiate on both sides of (27) and use (28), (33), and (34) to obtain, for $1 \leq i \leq n-1$,

$$
\begin{aligned}
& \dot{p}_{i}(t)=-\beta_{i} p_{i}(t-h)+\psi_{i}(t-h)-\psi_{i}(t)+p_{i+1}(t)-p_{i+1}(t-h) \\
& \dot{p}_{n}(t)=-\beta_{n} p_{n}(t-h)+\psi_{n}(t-h)-\psi_{n}(t)
\end{aligned}
$$

so, defining

$$
\begin{aligned}
& \Psi(s):=\left[\psi_{1}(s) \cdots \psi_{n}(s)\right]^{\top} \quad-c f . \text { Eq. (33) } \\
& \Phi(s):=\left[\begin{array}{ll}
-\psi_{1}(s)+p_{2}(s), \cdots-\psi_{n-1}(s)+p_{n}(s),-\psi_{n}(s)
\end{array}\right]^{\top},
\end{aligned}
$$

and

$$
B=\left[\begin{array}{ccccc}
0 & 1 & 0 & \cdots & 0 \\
& & & \ddots & \vdots \\
\vdots & & \ddots & & 0 \\
0 & & & & 1 \\
0 & & \cdots & & 0
\end{array}\right],
$$

we see that the closed-loop dynamics has the cascaded form

$$
\begin{aligned}
& \dot{e}(t)=-\alpha e(t-h)+B e(t)+\Psi(t) \\
& \dot{p}(t)=-\beta p(t-h)+\Phi(t)-\Phi(t-h)
\end{aligned}
$$

The rest of the proof relies on the sufficient conditions for the origin of the latter to be exponentially stable. These are presented in the next section where we formulate a self-contained and original statement for cascades of functional differential equations.

\section{Stability of CASCADED DELAY DIFFERENTIAL EQUATIONS}

Even though the stability problem for cascaded systems is well studied in the literature, we have been unable to locate an "off-theshelf" statement for cascades of functional differential equations like (35). Generally speaking, we consider the system

$$
\begin{aligned}
& \dot{z}_{1}(t)=-\alpha z_{1}(t-h)+B z_{1}(t)+\tilde{\Psi}(z(t)) \\
& \dot{z}_{2}(t)=-\beta z_{2}(t-h)+d_{1} \tilde{\Phi}(z(t))+d_{2} \tilde{\Phi}(z(t-h)),
\end{aligned}
$$

where $z_{1}, z_{2} \in \mathbb{R}^{n}, z=\left[z_{1}^{\top} z_{2}^{\top}\right]^{\top}, d_{1}, d_{2} \in \mathbb{R}$, and the functions $\tilde{\Psi}: \mathbb{R}^{2 n} \rightarrow \mathbb{R}^{n}$ and $\tilde{\Phi}: \mathbb{R}^{2 n} \rightarrow \mathbb{R}^{n}$ are continuous. 
Theorem 2 Consider the system (36). Assume that $\alpha$ and $\beta$ are diagonal positive matrices of dimension $n$, and that there exist $\gamma_{1}$, $\gamma_{2}>0$ such that

$$
|\tilde{\Psi}(z(s))| \leq \gamma_{1}\left|z_{2}(s)\right| \text { and }\left|d_{j} \tilde{\Phi}(z(s))\right| \leq \gamma_{2}\left|z_{2}(s)\right|,
$$

for $j \in\{1,2\}$. Denote by $b_{M}$ the spectral norm of $B$. Then, the origin is globally exponentially stable if (32) holds with $\gamma:=\max \left\{\gamma_{1}, \gamma_{2}\right\}$ and

$$
\alpha_{\min } \geq 4 b_{M}+\alpha_{\max }\left(\alpha_{\max }+2 b_{M}\right) h^{*} .
$$

Note that the Equations (35) have the form of (36) with $e=z_{1}$, $p=z_{2}, \alpha:=\operatorname{diag}\left\{\alpha_{1} \cdots \alpha_{n}\right\}, \beta:=\operatorname{diag}\left\{\beta_{1} \cdots \beta_{n}\right\}, d_{1}=1$, $d_{2}=-1, \Psi(s)=\tilde{\Psi}(z(s))$, and $\Phi(s)=\tilde{\Phi}(z(s))$. Therefore, the statement in Theorem 1 follows from Theorem 2 with $b_{M}=1$, $\gamma_{1}=\max \left\{\gamma_{i}\right\}$, where $\gamma_{i}$ are defined in (30) and $\gamma_{2}=\gamma_{1}+1$.

The proof of Theorem 2 is constructed based on a usual reasoning to establish stability for cascaded systems of ordinary differential equations. The exponential stability of the origin of the $z_{2}$-dynamics, (36b), is asserted by invoking the following output-injection statement which has its own interest; the proof is provided in the Appendix.

Lemma 1 (Output injection) Let $z \in \mathbb{R}^{n}$. The trivial solution of

$$
\dot{z}(t)=-\beta z(t-h)+d_{1} \Phi(t)+d_{2} \Phi(t-h), \quad d_{1}, d_{2} \in \mathbb{R},
$$

where $\beta \in \mathbb{R}^{n \times n}$ is diagonal positive definite, is globally exponentially stable if there exists $\gamma>0$ such that $\left|d_{j} \Phi(s)\right| \leq \gamma|z(s)|$, $j \in\{1,2\}$, and (32) holds.

Therefore, denoting by $\phi_{1}$ and $\phi_{2}$ the initial conditions of (36a) and (36b) respectively (see the Notation paragraph of Section I), we conclude that there exist $\kappa_{2}$ and $\lambda_{2}$ such that

$$
\left|z_{2}(t)\right| \leq \kappa_{2}\left\|\phi_{2}\right\| \mathrm{e}^{-\lambda_{2} t}
$$

A similar argument leads to the conclusion that the origin of (36a) without input, i.e., with $\tilde{\Psi}=0$, is exponentially stable. Indeed, since $\tilde{\Psi}=0$ if and only if, $z_{2}=0$ and $z_{2}(t)$ exponentially converges to zero, $\Psi(t)=\tilde{\Psi}(z(t))$ constitutes a vanishing perturbation to the $z_{1}$-dynamics, equivalently, the error dynamics (35a). Furthermore, in view of (40), $z_{2}$ is uniformly square integrable and bounded. Therefore, in view of (37), there exists $c_{\Psi}>0$ such that

$$
\max \left\{\sup _{t \geq-h}|\Psi(t)|, \quad\left(\int_{0}^{\infty}|\Psi(t)|^{2} d t\right)^{1 / 2}\right\} \leq c_{\Psi}\left\|\phi_{\Psi}\right\|
$$

where $\phi_{\Psi}:[0, h] \rightarrow \mathbb{R}^{n}$ and $\Psi:[-h, \infty) \rightarrow \mathbb{R}^{n}$ are absolutely continuous functions satisfying $\Psi(-s)=\phi(s)$ for all $s \in[0, h]$. Thus, by invoking Lemma 2 , given below, with $z=z_{1}$, we conclude that there exist $\kappa_{1}$ and $\lambda_{1}$ such that

$$
\left|z_{1}(t)\right| \leq \kappa_{1}\left[\left\|\phi_{1}\right\|+\left\|\phi_{2}\right\|\right] \mathrm{e}^{-\lambda_{1} t} .
$$

Lemma 2 (Vanishing perturbation) Consider the system

$$
\dot{z}(t)=-\alpha z(t-h)+B z(t)+\Psi(t), \quad z \in \mathbb{R}^{n}
$$

and assume that there exists $c_{\Psi}>0$ such that (41) holds. Then, there exist $\kappa, \lambda>0$ such that

$$
|z(t)| \leq \kappa\left[\left\|\phi_{z}\right\|+\left\|\phi_{\Psi}\right\|\right] e^{-\lambda t}
$$

where $\phi_{z}$ is the initial condition of $z$ in (42).

\section{CONCLUSiON}

In this paper, we proposed a new method for input delay compensation of restricted-feedback linearizable systems. Our approach consists in inverting the system, by computing the input that tracks asymptotically the desired output. In order to compute an estimate of the future of the system's state, instead of integrating the original system (which is nonlinear and might be unstable), we integrate the desired error dynamics (which is both linear and stable, at least asymptotically).

The main motivation of our work is to avoid, in the case of an unstable system, the pitfalls associated to predictors based on the integration of the system's dynamics (see, e.g., [4] and [5]). Of course, several other approaches have already been proposed in order to increase the robustness of predictors. The approach proposed in [19] is based in a similar idea, but its generalization in order to invert nonlinear systems does not seem straightforward. For the class of systems considered in this paper, another approach has been proposed in [7], [16], and [17], but using a more complex method.

We should nevertheless admit that, unlike those works (or [6]), we have not considered the problem of proving the stability of the numerical discretization of our control law. We have not considered neither the problem of non-constant input delays [12], nor the (natural) case of feedback-linearizable systems. This clearly indicates the preliminary nature of our work, and shows that much remains to be done to fully develop the proposed approach.

\section{APPENDIX}

\section{A. Proof of Lemma 1}

We denote by $\phi$ the initial condition of (39) as defined in the Notation paragraph of Section I. Let $z_{i}$ be the $i$ th element of $z \in$ $\mathbb{R}^{n}, \beta_{i}$ be the $i$ th element of the main diagonal of $\beta$, and let $\beta_{\max }$ be the largest of $\beta_{i}$ s. Consider the Lyapunov-Krasovskui functional $V: \mathbb{R} p \rightarrow \mathbb{R} p$

$$
\begin{aligned}
V(t):=V_{1}(t) & +V_{2}(t)+V_{3}(t)+V_{4}(t) \\
V_{1}(t) & :=\frac{1}{2} \sum_{i=1}^{n}\left[z_{i}(t)-\beta_{i} \int_{-h}^{0} z_{i}(t+\theta) d \theta\right]^{2} \\
V_{2}(t) & :=\frac{\beta_{\max }\left(\beta_{\max }+2 \gamma\right)}{2} \int_{-h}^{0} \int_{t+\theta}^{t}|z(s)|^{2} d s d \theta \\
V_{3}(t) & :=\frac{\gamma \beta_{\max }}{2}\left[\int_{-h}^{0}|z(t+\theta)| d \theta\right]^{2} \\
V_{4}(t) & :=\gamma \int_{t-h}^{t}|z(s)|^{2} d s,
\end{aligned}
$$

which satisfies the following properties. Let $\delta_{1}:=\beta_{\max }\left(\beta_{\max }+\right.$ $2 \gamma) / 2$ and $\delta_{2}:=\gamma \beta_{\max } / 2$, then

$$
\begin{aligned}
V(0) \leq & \frac{1}{2} \sum_{i=1}^{n}\left[z_{i}(0)-\beta_{i} \int_{-h}^{0} z_{i}(\theta) d \theta\right]^{2} \\
& +\delta_{1} \int_{-h}^{0} \int_{\theta}^{0}|z(s)|^{2} d s d \theta+\left(\delta_{2}+\gamma\right) \int_{-h}^{0}|z(s)|^{2} d s \\
\leq & |z(0)|^{2}+\frac{\left(\beta_{\max }^{2}+\delta_{1}\right) h^{2}+\left(\delta_{2}+\gamma\right) h}{2} \sup _{t \in[-h, 0]}|z(t)|^{2} \\
\leq & \max \left\{1, c_{\circ}\right\}\|\phi\|^{2}
\end{aligned}
$$

where

$$
c_{\circ}:=\frac{\left(\beta_{\max }^{2}+\delta_{1}\right) h^{2}+\left(\delta_{2}+\gamma\right) h}{2} .
$$


On the other hand, $V(t) \geq V_{1}(t)$ and

$$
\begin{aligned}
V_{1}(t) \geq & \frac{1}{2}|z(t)|^{2}-2 \sum_{i=1}^{n} \beta_{i}\left(\int_{-h}^{0} z_{i}(t+\theta) d \theta\right)^{2} \\
& \geq \frac{1}{2}|z(t)|^{2}-2 \beta_{\text {max }}^{2} h \sup _{t \in[-h, 0]}|z(t)|^{2}
\end{aligned}
$$

and we claim that, under (32),

$$
\dot{V}(t) \leq-\left(\beta_{\min } / 2\right)|z(t)|^{2} \leq 0
$$

therefore, using (45), (46), and $V(t) \leq V(0)$, we conclude that

$$
\sup _{t \geq-h}|z(t)| \leq 2 \max \left\{1, c_{\circ}+2 \beta_{\max }^{2} h\right\}\|\phi\| .
$$

Finally, we integrate on both sides of the first inequality in (47) to obtain

$$
\beta_{\min } \int_{0}^{t}|z(s)|^{2} d s \leq 2[V(0)-V(t)] \leq 2 V(0), \quad \forall t \geq 0
$$

hence

$$
\int_{0}^{\infty}|z(t)|^{2} d t \leq \frac{2 \max \left\{1, c_{\circ}\right\}}{\beta_{\min }}\|\phi\|^{2} .
$$

The result follows invoking the following statement which we adapted from [24, Lemma 3] for the purposes of this paper. Note that the converse statement of Lemma 3 is also true.

Lemma 3 Assume that there exist constants $r>0$ and $p \in[0, \infty)$ such that for each $h \in[0, \bar{h})$ there exist $c_{1}, c_{2}>0$ such that for all $t_{\circ} \in \mathbb{R}_{\geq 0}$, the function $t \mapsto z$ is defined on $\left[t_{\circ}-h, \infty\right)$ and satisfies

$$
\begin{aligned}
& \sup _{t \geq t_{\circ}-h}|z(t)| \leq c_{1}\|\phi\| \\
& \int_{t_{\circ}}^{\infty} z(t)^{p} d t \leq c_{2}\|\phi\|^{p}
\end{aligned}
$$

then, given $\epsilon \in(0,1)$,

$$
|z(t)| \leq c_{1}\|\phi\| e^{-\lambda\left(t-t_{\circ}\right)}
$$

where

$$
\lambda=\ln \left(\frac{\epsilon}{c_{1} c_{2}}\right)^{p} \ln \left(\frac{1}{\epsilon}\right)
$$

and $\phi(s)=z\left(t_{\circ}-s\right)$, for $s \in[0, h]$.

It is left to prove that (47) holds. The total derivative of $V$ along the trajectories of (39) satisfies

$$
\dot{V}(t) \leq Y_{1}(t)+Y_{2}(t)+Y_{3}(t)+Y_{4}(t)
$$

where

$$
\begin{aligned}
& Y_{1}(t):= {\left[z(t)-\beta \int_{-h}^{0} z(t+\theta) d \theta\right]^{\top} \times } \\
& {\left[-\beta z(t)+d_{1} \Phi(t)+d_{2} \Phi(t-h)\right] } \\
& Y_{2}(t):=-\delta_{1} \int_{-h}^{0}\left[|z(t)|^{2}+|z(t+\theta)|^{2}-2|z(t)|^{2}\right] d \theta \\
& Y_{3}(t):= \beta_{\max } \gamma \int_{-h}^{0}|z(t+\theta)| d \theta[|z(t)|-|z(t-h)|] \\
& Y_{4}(t):= \gamma\left[|z(t)|^{2}-|z(t-h)|^{2}\right] .
\end{aligned}
$$

To obtain $Y_{1}$ we have used

$$
\begin{gathered}
\frac{d}{d t}\left[z_{i}(t)-\beta_{i} \int_{-h}^{0} z_{i}(t+\theta) d \theta\right]=\dot{z}_{i}(t)-\beta_{i} \int_{-h}^{0} \dot{z}_{i}(t+\theta) d \theta \\
=-\beta_{i} z_{i}(t-h)+\beta_{i} z_{i}(t)-\beta_{i} z_{i}(t)+d_{1} \Phi(t) \\
\quad+d_{2} \Phi(t-h)-\beta_{i} \int_{-h}^{0} \dot{z}_{i}(t+\theta) d \theta \\
=\beta_{i} \int_{t-h}^{t} \dot{z}_{i}(s) d s-\beta_{i} z_{i}(t)+d_{1} \Phi(t) \\
\quad+d_{2} \Phi(t-h)-\beta_{i} \int_{t-h}^{t} \dot{z}_{i}(s) d s
\end{gathered}
$$

where, for the last term we used the identity

$$
\int_{-h}^{0} w(t+\theta) d \theta=\int_{t-h}^{t} w(\theta) d \theta, \quad \forall t \geq 0 .
$$

To obtain $Y_{2}$, we have used

$$
\begin{aligned}
\dot{V}_{2}(t):= & \frac{\beta_{\max }\left(\beta_{\max }+2 \gamma\right)}{2} \int_{-h}^{0} \frac{d}{d t} \int_{t+\theta}^{t}|z(s)|^{2} d s d \theta \\
& =\frac{\beta_{\max }\left(\beta_{\max }+2 \gamma\right)}{2} \int_{-h}^{0}\left[|z(t)|^{2}-|z(t+\theta)|^{2}\right] d \theta .
\end{aligned}
$$

For the computation of $Y_{3}$, which satisfies $Y_{3} \geq \dot{V}_{3}$, we used (50) and

$$
\left[\int_{-h}^{0} z_{i}(t+\theta) d \theta\right]\left[z_{i}(t)\right]=\int_{t-h}^{t} z_{i}(\theta) z_{i}(t) d \theta
$$

Finally, $Y_{4}$ is obtained by a direct computation of $\dot{V}_{4}$ which leads to $\dot{V}_{4}=Y_{4}$. Now,

$$
\begin{aligned}
& Y_{1}(t)+Y_{2}(t) \leq-\beta_{\min }|z(t)|^{2}+\gamma|z(t)|^{2} \\
& +\gamma|z(t)||z(t-h)|+\beta_{\max }\left(\beta_{\max }+2 \gamma\right) h|z(t)|^{2} \\
& +\beta_{\max }^{2} \int_{-h}^{0}|z(t+\theta)| d \theta|z(t)| \\
& \quad+\beta_{\max } \gamma \int_{-h}^{0}|z(t+\theta)| d \theta[|z(t)|+|z(t-h)|] \\
& \quad-\frac{\beta_{\max }\left(\beta_{\max }+2 \gamma\right)}{2} \int_{-h}^{0}\left[|z(t)|^{2}+|z(t+\theta)|^{2}\right] d \theta
\end{aligned}
$$

hence

$$
\begin{gathered}
Y_{1}(t)+Y_{2}(t)+Y_{3}(t) \leq-\left[\beta_{\min }-\gamma-\beta_{\max }\left(\beta_{\max }+2 \gamma\right) h\right]|z(t)|^{2} \\
+\gamma|z(t)||z(t-h)|+\beta_{\max }^{2} \int_{-h}^{0}|z(t+\theta)| d \theta|z(t)| \\
+2 \beta_{\max } \gamma \int_{-h}^{0}|z(t+\theta)| d \theta|z(t)| \\
\quad-\frac{\beta_{\max }\left(\beta_{\max }+2 \gamma\right)}{2} \int_{-h}^{0}\left[|z(t)|^{2}+|z(t+\theta)|^{2}\right] d \theta
\end{gathered}
$$

and the last three terms equal to

$$
-\delta_{1} \int_{-h}^{0}\left[|z(t)|^{2}-2|z(t)||z(t+\theta)|+|z(t+\theta)|^{2}\right] d \theta \leq 0 .
$$

Thus,

$$
\dot{V}(t) \leq-\left[\begin{array}{c}
|z(t)| \\
|z(t-h)|
\end{array}\right]^{\top}\left[\begin{array}{cc}
\beta_{\min }-2 \gamma+\delta_{1} h & \gamma / 2 \\
\gamma / 2 & \gamma
\end{array}\right]\left[\begin{array}{c}
|z(t)| \\
|z(t-h)|
\end{array}\right]
$$

and in view of (32), the matrix above is positive definite and $2 \dot{V}(t) \leq$ $-\beta_{\text {min }}|z(t)|^{2}-\gamma|z(t-h)|^{2}$. 


\section{B. Proof of Lemma 2}

Equation (42) with $\Psi \equiv 0$ has the form (39) with $\beta=\alpha, d_{1}=$ $1, d_{2}=0$ and $\Phi(t):=B z(t)$ therefore, Lemma 1 applies with $\gamma=b_{M} \geq|B|$. Moreover, by assumption, $\Psi(t)$ converges to 0 exponentially fast -see Lemma 3 , therefore it is only left to prove that the nominal system corresponding to (42) conserves the property of (uniform) exponential stability, under the (uniformly) vanishing perturbation $\Psi$. For this, we use again the function $V$ defined in (44) with $\beta=\alpha$ which satisfies, along the trajectories of (42),

$$
\dot{V}(t)=\sum_{i=1}^{4} Y_{i}(t)+\left[z(t)-\alpha \int_{-h}^{0} z(t+\theta) d \theta\right]^{\top} \Psi(t)
$$

hence, from (47), we have

$$
\dot{V}(t) \leq-\left(\alpha_{\text {min }} / 2\right)|z(t)|^{2}+\left[z(t)-\alpha \int_{t-h}^{t} z(\theta) d \theta\right]^{\top} \Psi(t),
$$

where $\alpha$ satisfies (38). To show that the solutions are bounded we proceed by contradiction. If $|z(t)| \rightarrow \infty$ as $t \rightarrow \infty$ then $|z(t)| \geq$ $|z(t-h)|$ and for all $t \geq 0$,

$$
\dot{V}(t) \leq-\left(\alpha_{\min } / 2\right)|z(t)|^{2}+\left(1+\alpha_{\max } h\right)|z(t)||\Psi(t)| .
$$

Since $|\Psi(t)| \rightarrow 0$ let $t_{\Psi}^{\eta}$ be the smallest $t$ such that $|\Psi(t)| \leq \eta$ for all $t \geq t_{\Psi}^{\eta}$ and any $\eta>0$ then, for all $t \geq t_{\Psi}^{\eta}$

$$
\dot{V}(t) \leq-\left[\left(\alpha_{\min } / 2\right)|z(t)|-\eta\left(1+\alpha_{\max } h\right)\right]|z(t)| .
$$

Now let $t_{z}^{1}$ be the smallest $t$ such that $|z(t)| \geq 1$ then, for all $t \geq$ $\max \left\{t_{z}^{1}, t_{\Psi}^{\eta}\right\}$,

$$
\dot{V}(t) \leq-\left[\left(\alpha_{\min } / 2\right)-\eta\left(1+\alpha_{\max } h\right)\right]|z(t)| .
$$

Let $\eta \leq \eta^{*}$ with

$$
\eta^{*}:=\frac{\alpha_{\min }}{4\left(1+\alpha_{\max } h\right)}
$$

then

$$
\dot{V}(t) \leq-\frac{\alpha_{\min }}{4}|z(t)| \quad \forall t \geq \max \left\{t_{z}^{1}, t_{\Psi}^{\eta}\right\}
$$

that is, $V(t) \leq 0$ for all $t \geq \max \left\{t_{z}^{1}, t_{\Psi}^{\eta}\right\}$ and any $\eta \in\left(0, \eta^{*}\right]$ which implies that $V(t) \leq V(0)$ and in turn, (48) holds with an appropriate redefinition of the constant $c_{\circ}$ and $\beta_{\max }=\alpha_{\max }$. That is, the solutions are bounded.

Furthermore, again from (51), we have

$$
\dot{V}(t) \leq-\left(\alpha_{\min } / 2\right)|z(t)|^{2}+\lambda_{1}\left(1+\alpha_{\max } h\right)^{2}|z(t)|^{2}+\frac{|\Psi(t)|^{2}}{\lambda_{1}}
$$

for any $\lambda_{1}>0$ therefore,

$$
\dot{V}(t) \leq-c|z(t)|^{2}+\frac{|\Psi(t)|^{2}}{\lambda_{1}}
$$

where

$$
c:=\left[\left(\alpha_{\min } / 2\right)-\lambda_{1}^{2}\left(1+\alpha_{\max } h\right)^{2}\right]
$$

is positive for a suitable choice of $\lambda_{1}$. Therefore,

$$
\int_{0}^{\infty}|z(t)|^{2} d t \leq \frac{1}{c}\left[V(0)+\frac{1}{\lambda_{1}} \int_{0}^{\infty}|\Psi(t)|^{2} d t\right] .
$$

where $V(0)$ satisfies (45). The result follows using (41) and invoking Lemma 3.

\section{REFERENCES}

[1] O. Smith, "A controller to overcome dead time," ISA Journal, vol. 6, no. 2 , pp. $28-33,1959$.
[2] A. Manitius and A. Olbrot, "Finite spectrum assignment problem for systems with delays," IEEE Transactions on Automatic Control, vol. 24, no. 4, pp. 541-552, 1979.

[3] Z. Artstein, "Linear systems with delayed controls: A reduction," IEEE Transactions on Automatic Control, vol. 27, no. 4, pp. 869-879, 1982.

[4] K. Engelborghs, M. Dambrine, and D. Roose, "Limitations of a class of stabilization methods for delay systems," IEEE Transactions on Automatic Control, vol. 46, no. 2, pp. 336-339, 2001.

[5] S. Mondié and W. Michiels, "Finite spectrum assignment of unstable time-delay systems with a safe implementation," IEEE Transactions on Automatic Control, vol. 48, no. 12, pp. 2207-2212, 2003.

[6] F. Mazenc and D. Normand-Cyrot, "Stabilization of linear input delayed dynamics under sampling," in Proceedings of the IEEE Conference on Decision and Control, (Hawaii (USA)), pp. 7523-7528, 2012.

[7] I. Karafyllis, "Stabilization by means of approximate predictors for systems with delayed input," SIAM J. of Control and Optimization, vol. 49, no. 3, pp. 1100-1123, 2011.

[8] F. Mazenc and P. Bliman, "Backstepping design for time-delay nonlinear systems," IEEE Transactions on Automatic Control, vol. 51, no. 1, pp. 149-154, 2006.

[9] M. Krstic, "Input delay compensation for forward complete and strictfeedforward nonlinear systems," IEEE Transactions on Automatic Control, vol. 55, no. 2, pp. 287-303, 2010.

[10] F. Mazenc, S. Niculescu, and M. Bekaik, "Stabilization of nonlinear systems with delay in the input through backstepping," in Proceedings of the IEEE Conference on Decision and Control and the European Control Conference, (Orlando (USA)), pp. 7605-7610, 2011.

[11] M. Koo, H. Choi, and J. Lim, "Global regulation of a class of feedforward and non-feedforward nonlinear systems with a delay in the input," Automatica, vol. 48, no. 10, pp. 2607-2613, 2012.

[12] N. Bekiaris-Liberis and M. Krstic, "Compensation of state-dependent input delay for nonlinear systems," Automatic Control, IEEE Transactions on, vol. 58, no. 2, pp. 275-289, 2013.

[13] R. Brockett, "Feedback invariants for nonlinear systems," in Proceedings of the IFAC World Congress, (Helsinki (Finland)), 1979.

[14] W. Dayawansa, W. Boothby, and D. Elliott, "Global state and feedback equivalence of nonlinear systems," Systems and Control Letters, vol. 6, no. 4, pp. 229-234, 1985.

[15] W. Respondek, "Global aspects of linearization, equivalence to polynomial forms and decomposition of nonlinear control systems," in Algebraic and Geometric Methods in Nonlinear Control Theory, pp. 257284, Springer, 1986.

[16] I. Karafyllis and M. Krstic, "Stabilization of nonlinear delay systems using approximate predictors and high-gain observers," Automatica, vol. 49, no. 12, pp. 3623-3631, 2013.

[17] I. Karafyllis and M. Krstic, "Numerical schemes for nonlinear predictor feedback," Mathematics of Control, Signals, and Systems, vol. 26, no. 4, pp. 519-546, 2014.

[18] I. Karafyllis, M. Krstic, T. Ahmed-Ali, and F. Lamnabhi-Lagarrigue, "Global stabilisation of nonlinear delay systems with a compact absorbing set," International Journal of Control, vol. 87, no. 5, pp. 1010-1027, 2014.

[19] G. Besançon, D. Georges, and Z. Benayache, "Asymptotic state prediction for continuous-time systems with delayed input and application to control," in Proceedings of the European Control Conference, (Kos (Greece)), 2007.

[20] A. Ramírez, S. Mondié, and R. Garrido, "Proportional integral retarded control of second order linear systems," in IEEE Conference on Decision and Control, pp. 2239-2244, IEEE, 2013.

[21] H. Estrada-García, O. Penaloza-Mejia, C. Moog, and L. MárquezMartínez, "Trajectory tracking problem: causal solutions for non-linear time-delay systems," International Journal of Control, vol. 81, no. 8, pp. 1202-1209, 2008.

[22] B. Cahlon and D. Schmidt, "Stability criteria for certain high odd order delay differential equations," Journal of Computational and Applied Mathematics, vol. 200, no. 1, pp. 408-423, 2007.

[23] B. Cahlon and D. Schmidt, "Stability criteria for certain high even order delay differential equations," Journal of Mathematical Analysis and Applications, vol. 334, no. 2, pp. 859-875, 2007.

[24] E. Panteley, A. Loría, and A. Teel, "Relaxed persistency of excitation for uniform asymptotic stability," IEEE Transactions on Automatic Control, vol. 46, no. 12, pp. 1874-1886, 2001. 